\title{
In vitro activity of tigecycline in combination with various antimicrobials against multidrug resistant Acinetobacter baumannii Luigi Principe $^{1}$, Silvia D'Arezzo ${ }^{1}$, Alessandro Capone ${ }^{1}$, Nicola Petrosillo ${ }^{1}$ and Paolo Visca*1,2
}

\author{
Address: ${ }^{1}$ National Institute for Infectious Diseases "Lazzaro Spallanzani", Via Portuense 292, 00149 Rome, Italy and ${ }^{2}$ Department of Biology, \\ University Roma Tre, Viale Marconi 446, 00146 Rome, Italy \\ Email: Luigi Principe - luigi.principe@inmi.it; Silvia D'Arezzo - darezzo@inmi.it; Alessandro Capone - capone@inmi.it; \\ Nicola Petrosillo - petrosillo@inmi.it; Paolo Visca* - visca@uniroma3.it \\ * Corresponding author
}

Published: 21 May 2009

Annals of Clinical Microbiology and Antimicrobials 2009, 8:18 doi:10.1 186/1476-07II-8-18

This article is available from: http://www.ann-clinmicrob.com/content/8/1/18

(c) 2009 Principe et al; licensee BioMed Central Ltd.

This is an Open Access article distributed under the terms of the Creative Commons Attribution License (http://creativecommons.org/licenses/by/2.0), which permits unrestricted use, distribution, and reproduction in any medium, provided the original work is properly cited.
Received: 24 February 2009

Accepted: 21 May 2009

\begin{abstract}
Background: Infections sustained by multidrug-resistant (MDR) and pan-resistant Acinetobacter baumannii have become a challenging problem in Intensive Care Units. Tigecycline provided new hope for the treatment of MDR A. baumannii infections, but isolates showing reduced susceptibility have emerged in many countries, further limiting the therapeutic options. Empirical combination therapy has become a common practice to treat patients infected with MDR A. baumannii, in spite of the limited microbiological and clinical evidence supporting its efficacy. Here, the in vitro interaction of tigecycline with seven commonly used anti-Acinetobacter drugs has been assessed.
\end{abstract}

Methods: Twenty-two MDR A. baumannii isolates from Intensive Care Unit (ICU) patients and two reference strains for the European clonal lineages I and II (including 3, I5 and 6 isolates that were resistant, intermediate and susceptible to tigecycline, respectively) were tested. Antimicrobial agents were: tigecycline, levofloxacin, piperacillin-tazobactam, amikacin, imipenem, rifampicin, ampicillin-sulbactam, and colistin. MICs were determined by the broth microdilution method. Antibiotic interactions were determined by chequerboard and time-kill assays. Only antibiotic combinations showing synergism or antagonism in both chequerboard and time-kill assays were accepted as authentic synergistic or antagonistic interactions, respectively.

Results: Considering all antimicrobials in combination with tigecycline, chequerboard analysis showed $5.9 \%$ synergy, $85.7 \%$ indifference, and $8.3 \%$ antagonism. Tigecycline showed synergism with levofloxacin (4 strains; 16.6\%), amikacin ( 2 strains; $8.3 \%$ ), imipenem ( 2 strains; $8.3 \%$ ) and colistin (2 strains; $8.3 \%$ ). Antagonism was observed for the tigecycline/piperacillin-tazobactam combination (8 strains; $33.3 \%$ ). Synergism was detected only among tigecycline non-susceptible strains. Time-kill assays confirmed the synergistic interaction between tigecycline and levofloxacin, amikacin, imipenem and colistin for 5 of 7 selected isolates. No antagonism was confirmed by time-kill assays.

Conclusion: This study demonstrates the in vitro synergistic activity of tigecycline in combination with colistin, levofloxacin, amikacin and imipenem against five tigecycline non-susceptible $A$. baumannii strains, opening the way to a more rationale clinical assessment of novel combination therapies to combat infections caused by MDR and pan-resistant A. baumannii. 


\section{Background}

Acinetobacter baumannii has emerged as a leading nosocomial pathogen, particularly in Intensive Care Units (ICUs), where several outbreaks have been described [1]. The epidemic potential and the clinical severity of $A$. baumannii infections are primarily related to the propensity of this organism to develop resistance to a variety of antimicrobial agents, including broad-spectrum beta-lactams, aminoglycosides, fluoroquinolones and carbapenems [2].

Carbapenems remain drugs of choice for the treatment of A. baumannii infection, but their efficacy can be compromised by the spread of novel class $D$ carbapenemases $[3,4]$. As a result, carbapenem-intermediate or -resistant $A$. baumannii isolates are becoming increasingly prevalent in several countries [5]. Colistin, an old antibiotic from the polymixin group, is very effective against multidrug-resistant (MDR) A. baumannii isolates, but the emergence of resistance has occasionally been experienced [6]. Moreover, unfavourable pharmacokinetic properties and possible adverse effects restrict its clinical use $[7,8]$. Tigecycline, a new semi-synthetic tetracycline, has provided hope for the treatment of A. baumannii infections, including carbapenem-resistant isolates $[9,10]$. However, A. baumannii isolates showing reduced susceptibility to tigecycline have recently been identified in Israel [11], Spain [12], Italy [13], USA [14], and China [15]. Moreover, the exposure to sub-MIC tigecycline concentrations has been shown to facilitate the development of resistance in A. baumannii both in vitro and in vivo [16-18]. In this scenario, combination therapy has become the ultimate resource to treat MDR and pan-resistant A. baumannii infections [19], but its actual efficacy is unclear from a microbiological and clinical viewpoint. Previous investigations revealed an overall indifferent effect of tigecycline in combination with other antimicrobial agents commonly used against Acinetobacter spp., including carbapenems, fluoroquinolones, rifampicin, ampicillin-sulbactam, piperacillintazobactam, polymyxin B and colistin [20-22]. Only few clinical studies reported successful in vivo treatment of MDR A. baumannii infection with tigecycline in combination with colistin, meropenem, piperacillin-tazobactam and cotrimoxazole $[23,24]$. However, these results were not supported by an in vitro synergy study. Recently, tigecycline/amikacin synergistic interactions have been observed in vitro [22,25], but rarely in case of carbapenemresistant $A$. baumannii strains [25].

The aim of this study was to investigate the in vitro activity of tigecycline in combination with a variety of commonly used antimicrobial agents against MDR A. baumannii isolates, including tigecycline intermediate and resistant strains. Part of this work has been presented at the 48th ICAAC meeting, Washington DC (USA), 2008 (Poster C13817).

\section{Methods \\ Bacterial isolates and epidemiological typing}

Twenty-two A. baumannii isolates were selected from a well-characterized set of MDR A. baumannii isolates collected in the period January 2004-June 2005 from the ICU of 7 general hospitals of the Rome (Italy) urban area $[13,26]$. Twenty A. baumannii isolates were obtained from clinical specimens of ICU patients, and 2 were recovered from the ICU environment (Table 1). None of the patients underwent previous treatment with tigecycline. The collection also comprises an index strain (study code 115, also called ACICU) from an ICU outbreak [27], whose complete genome has recently been sequenced [28]. The two prototypic strains for the epidemic European clonal lineages I (RUH875) and II (RUH134) were included as reference [29]. The selection criteria for A. baumannii isolates were based on hospital of origin (representing 7 hospitals, named A to $G$, in the Rome urban area), tigecycline susceptibility (6 sensitive; 15 intermediate; 3 resistant), molecular type (RAPD fingerprint, pulsotype and sequence group as described) [26] and antibiotic resistance profile (Table 1 ). Since there is no widely accepted definition for MDR A. baumannii, [30], hereafter we shall refer to the MDR phenotype as diminished susceptibility to $\geq 2$ of the following drug classes: antipseudomonal cephalosporins, antipseudomonal carbapenems, $\beta$ lactam- $\beta$-lactamase inhibitor combinations, antipseudomonal fluoroquinolones and aminoglycosides, according to ref. [31]. Twenty-one isolates were genetically related to either the European clonal lineage I ( 6 isolates) or II (15 isolates), and indicated as type 2 or 1 , respectively, upon sequence group analysis, RAPD, and pulsotyping. Typing data have been published elsewhere [26]. In addition, 3 A. baumannii isolates (study codes 50, 75 and 105) showing a variant molecular type were included. Isolates belonging to RAPD types $1,1 \mathrm{a}$ and 4 were resistant to carbapenems, while those belonging to RAPD types 2 and 2 a were susceptible (Table 1 ).

\section{Antimicrobial agents and MIC assays}

Antimicrobial agents were: levofloxacin, piperacillin-tazobactam, amikacin, imipenem, rifampicin, ampicillin-sulbactam, colistin, and tigecycline. MIC determinations for all antibiotics were performed by the broth microdilution method, according to the Clinical and Laboratory Standards Institute (CLSI) protocol [32]. All powders were obtained from the Sigma-Aldrich (Milan, Italy), except tigecycline (Wyeth-Ayerst, Collegeville, Pennsylvania, USA). MICs were determined in 96-well microtiter plates (Costar, Cambridge, Massachusetts, USA) containing freshly prepared Mueller-Hinton broth (Oxoid, Milan, Italy), to prevent oxidative degradation of tigecycline in aqueous solution (Wyeth Research, unpublished data). The inoculum was adjusted to $\sim 5 \times 10^{5} \mathrm{CFU} / \mathrm{ml}$ in a 100 $\mu \mathrm{l}$ final volume, and microtiter plates were visually read 
Table I: Characteristics of $A$. baumannii isolates ${ }^{a}$

\begin{tabular}{|c|c|c|c|c|c|}
\hline $\begin{array}{l}\text { Study code } \\
\text { (Hospital) }\end{array}$ & Source (isolation date) & Sequence group & RAPD type & Pulsotype & Antibiotic resistance profile ${ }^{b}$ \\
\hline $5(A)$ & Respiratory secretions (06/15/04) & I & I & $\mathrm{I}$ & LVX TZP AMK IPM RIF SAM TIG \\
\hline II (B) & Respiratory secretions (05/I5/04) & 1 & I & I & LVX TZP AMK IPM RIF TIG \\
\hline $16(B)$ & Respiratory secretions $(06 / 21 / 04)$ & I & I & I & LVX TZP AMK IPM TIG \\
\hline $28(B)$ & Environmental, laryngoscope $(06 / 21 / 04)$ & I & I & I & LVX TZP AMK IPM RIF TIG \\
\hline $29(B)$ & Central venous catheter $(07 / 19 / 04)$ & 1 & I & I & LVX TZP AMK IPM RIF TIG \\
\hline $32(\mathrm{~B})$ & Respiratory secretions (07//3/04) & I & I & I & LVX TZP AMK IPM RIF TIG \\
\hline $50(C)$ & Respiratory secretions (0I/07/04) & 4 & Ia & I & LVX TZP AMK IPM RIF SAM CS \\
\hline $62(C)$ & Wound swab (03/19/04) & 2 & 2 & 2 & LVX TZP RIF TIG \\
\hline $63(C)$ & Respiratory secretions $(06 / 21 / 04)$ & I & I & I & LVX TZP AMK IPM SAM TIG \\
\hline $71(C)$ & Environmental, desk surface $(07 / 26 / 04)$ & I & I & I & LVX TZP AMK IPM SAM TIG \\
\hline $73(\mathrm{E})$ & Respiratory secretions $(05 / 28 / 05)$ & I & I & I & LVX TZP AMK IPM RIF \\
\hline $75(C)$ & Wound swab $(05 / 17 / 05)$ & 2 & $2 \mathrm{a}$ & 2 & LVX TZP AMK RIF TIG \\
\hline 80 (D) & Wound swab $(01 / 24 / 05)$ & I & I & I & LVX TZP AMK IPM RIF SAM TIG \\
\hline $82(\mathrm{D})$ & Wound swab (04/12/04) & 2 & 2 & 2 & LVX TZP RIF TIG \\
\hline 86 (D) & Urine $(04 / 11 / 04)$ & I & I & I & LVX TZP AMK IPM RIF TIG \\
\hline 87 (D) & Central venous catheter $(07 / 06 / 04)$ & 2 & 2 & 2 & LVX TZP AMK TIG \\
\hline 88 (D) & Respiratory secretions $(03 / 02 / 04)$ & 2 & 2 & 2 & LVX TZP RIF TIG \\
\hline 89 (D) & Respiratory secretions $(02 / 1 \mathrm{I} / 05)$ & 2 & 2 & 2 & LVX TZP RIF TIG \\
\hline 93 (D) & Central venous catheter $(10 / 04 / 04)$ & I & I & I & LVX TZP AMK IPM SAM TIG \\
\hline $100(\mathrm{~F})$ & Respiratory secretions $(03 / 01 / 05)$ & I & 1 & 1 & LVX TZP AMK IPM RIF SAM TIG \\
\hline $105(C)$ & Cerebrospinal fluid $(06 / 27 / 05)$ & Variant & 4 & 3 & LVX TZP AMK RIF SAM \\
\hline $115(\mathrm{G}) \mathrm{c}$ & Blood culture $(06 / 10 / 05)$ & 1 & i & I & LVX TZP AMK IPM RIF SAM \\
\hline RUH I34d & Urine (1982) & 2 & I & 1 & SAM \\
\hline RUH $875^{d}$ & Urine (1984) & I & 2 & 2 & SAM RIF \\
\hline
\end{tabular}

a Data are from refs [13,26].

$\mathrm{b}$ Isolates showing an intermediate level of susceptibility were classified as resistant.

c $A$. baumannii index strain, also called ACICU $[27,28]$.

d Representative of the European clonal lineages I (RUH 875) and II (RUH 134) [29].

after incubation for $24 \mathrm{~h}$ at $37^{\circ} \mathrm{C}$. Escherichia coli ATCC 25922 and Staphylococcus aureus ATCC 29213 were used as internal quality control strains. The US FDA breakpoints approved for Enterobacteriaceae were applied to define tigecycline susceptibility (susceptibility, $\leq 2 \mathrm{mg} / \mathrm{L}$; resistance, $\geq 8 \mathrm{mg} / \mathrm{L}$ ). MIC results were interpreted according to the CLSI breakpoint criteria [32]. The criteria proposed by Gales et al were used for interpretation of colistin susceptibility [33]. Breakpoints for rifampicin were interpreted according to Hogg et al [34].

\section{Chequerboard assay}

Antibiotic interactions were determined using the chequerboard assay as previously described [22]. The range of drug concentration used in the chequerboard analysis was such that the dilution range encompassed the MIC for each drug used in the analysis. Broth microdilution plates were inoculated with each A. baumannii isolate to yield $\sim 5$ $\times 10^{5} \mathrm{CFU} / \mathrm{ml}$ in a $100-\mu \mathrm{l}$ final volume, and incubated for $18 \mathrm{~h}$ at $37^{\circ} \mathrm{C}$.

Synergy has been defined as requiring a fourfold reduction in the MIC of both antibiotics in combination, compared with each used alone, measuring the fractional inhibitory concentration index (FICI). The FICI was calcu- lated for each combination using the following formula: $\mathrm{FICI}=\mathrm{FIC}_{\mathrm{A}}+\mathrm{FIC}_{\mathrm{B}}$, where $\mathrm{FIC}_{\mathrm{A}}=$ MIC of drug A in combination/MIC of drug $\mathrm{A}$ alone, and $\mathrm{FIC}_{\mathrm{B}}=\mathrm{MIC}$ of drug $\mathrm{B}$ in combination/MIC of drug $\mathrm{B}$ alone. The FICI was interpreted as follows: synergy, FICI $\leq 0.5$; indifference, $0.5<$ $\mathrm{FICI} \leq 4$; antagonism, FICI $>4$ [22].

\section{Time-kill assays}

Tubes containing freshly prepared Mueller-Hinton broth supplemented with the drug were inoculated with A. baumannii isolates to a density of $\sim 5 \times 10^{5} \mathrm{CFU} / \mathrm{ml}$ in a final volume of $10 \mathrm{ml}$ and incubated in a shaking bath at $37^{\circ} \mathrm{C}$. Aliquots were removed at time $0,3,6$, and $24 \mathrm{~h}$ post-inoculation, and serially diluted in saline for determination of viable counts. Diluted samples $(100 \mu \mathrm{l})$ were plated on Mueller Hinton agar plates and bacterial counts were determined after 18 -h incubation at $37^{\circ} \mathrm{C}$. The antibiotic concentrations used in time-kill assays corresponded to $0.5-, 1-$, and 2 -fold the MIC values in combination as determined by the chequerboard method, i.e. 2- to 16fold lower than the MIC of each antibiotic alone (see Results). The bactericidal activity was defined as $=3 \log _{10}$ $\mathrm{CFU} / \mathrm{ml}$ reduction in the colony count relative to the initial inoculum [21]. Synergy was interpreted as $\geq 2 \log _{10}$ decrease in CFU/ml by the drug combination when com- 
pared with its most active constituent, and $=2 \log _{10}$ decrease in the $\mathrm{CFU} / \mathrm{ml}$ below the initial inoculum, at any time point. The drug combination was considered to be antagonistic for $=2 \log _{10}$ increase in CFU $/ \mathrm{ml}$ and indifferent for $<2 \log _{10}$ change in CFU/ml [22]. All synergistic interactions were confirmed by triplicate assays. Only antibiotic combinations showing synergism or antagonism in both chequerboard and time-kill assays were accepted as authentic synergistic or antagonistic interactions, respectively.

\section{Determination of mutation frequencies for resistance to antibiotics}

This was performed essentially as described by Miller $e$ al . [35]. Approximately $10^{8}$ cells from overnight cultures in Mueller-Hinton broth were spread onto triplicate Mueller-Hinton agar plates supplemented with the selective antibiotic at a concentration that was four-fold higher than the respective MIC for an individual isolate. After 48 $\mathrm{h}$ incubation at $37^{\circ} \mathrm{C}$, the number of colonies was counted, and mutation frequencies were expressed as the mean number of colonies recovered as a fraction of total viable bacteria plated. Isolates with mutation rates $>10^{-7}$ were considered to be mutators [36].

\section{Detection of ade genes for active efflux systems}

The presence of $a d e B$, adeJ, adeE, and $a d e Y$, and of the twocomponent regulatory system adeRS which controls Ade$A B C$ expression was investigated by PCR as previously reported [37-39]. The identity of $a d e B$, adeJ, adeR, and adeS amplicons was confirmed by direct DNA sequencing.

\section{Results}

The antibiotic susceptibility levels, expressed as MIC of levofloxacin, piperacillin-tazobactam, amikacin, imipenem, rifampicin, ampicillin-sulbactam, colistin and tigecycline, were preliminarily determined for the whole panel of 24 A. baumannii isolates [see Additional file 1]. All isolates, except the prototypic strains for the European clonal lineages I (RUH 875) and II (RUH 134), were resistant to levofloxacin and piperacillin-tazobactam (22 isolates each, 91.7\%). A high percentage of isolates were resistant to amikacin (18 isolates, 75.0\%), imipenem (15 isolates, $62.5 \%$ ), and rifampicin (18 isolates, $75.0 \%)$, while only $9(37.5 \%)$ and $1(4.2 \%)$ isolates were resistant to ampicillin-sulbactam and colistin, respectively. Eighteen isolates $(75.0 \%)$ were non-susceptible to tigecycline, including both resistant and intermediate phenotypes. Reference strains RUH 875 and RUH 134, isolated in early 1980s, showed an overall susceptible profile (Tables 1 and Additional file 1).

Chequerboard analysis performed with all antimicrobials in combination with tigecycline showed 5.9\% synergy, $85.7 \%$ indifference, and $8.3 \%$ antagonism (Table 2). Tige- cycline exerted synergistic activity with levofloxacin (4 isolates), amikacin, imipenem and colistin (2 isolates each). Notably, synergistic effects were observed only among tigecycline non-susceptible isolates (Table 2). Antagonistic interactions were frequently observed for tigecycline/ piperacillin-tazobactam (8 isolates), and to a lesser extent for tigecycline/amikacin (3 isolates), tigecycline/colistin, tigecycline/ampicillin-sulbactam and tigecycline/ rifampicin ( 1 isolate each) (Table 2). The concentration of individual drugs in synergistic combinations is shown in Table 3.

All synergistic interactions inferred from chequerboard analysis were reassessed by time-kill kinetic experiments performed with tigecycline in combination with levofloxacin, amikacin, imipenem and colistin. Time-kill diagrams for effective combinations are shown in Figure 1. Five of 10 synergistic combinations, namely tigecycline/ levofloxacin (2 out of 4 isolates), tigecycline/amikacin, tigecycline/imipenem and tigecycline/colistin (1 out of 2 isolates each) were confirmed. Synergistic effects were observed at $3 \mathrm{~h}$ for tigecycline/colistin $(2 / 0.25 \mathrm{mg} / \mathrm{L}=1$ / 2 MIC for both antibiotics), at $6 \mathrm{~h}$ for tigecycline/imipenem $(0.5 / 16 \mathrm{mg} / \mathrm{L}=1 / 2$ MIC for tigecycline and $1 / 8$ MIC for imipenem) and tigecycline/levofloxacin (0.25/4 $\mathrm{mg} / \mathrm{L}=1 / 16 \mathrm{MIC}$ for tigecycline and 1/4 MIC for levofloxacin), and at $24 \mathrm{~h}$ for tigecycline/amikacin $(1 / 64 \mathrm{mg} /$ $\mathrm{L}=1 / 4$ MIC for both antibiotics). Re-growth was observed after $24 \mathrm{~h}$ for tigecycline/colistin, tigecycline/imipenem and tigecycline/levofloxacin combinations (Figure 1). No synergistic combination resulted in bactericidal activity. Moreover, none of the 14 antagonistic interactions inferred from chequerboard analysis was confirmed by time-kill assays (data not shown).

To determine if regrowth in time-kill assays was due to $A$. baumannii hypermutability, the spontaneous mutation frequency toward antibiotic resistance was determined for isolates showing regrowth at $24 \mathrm{~h}$ (study codes $5,11,75$, 80 ) or not (study code 71). Resistant mutants were not detected (mutation rate $<10^{-8}$ ) upon selection with levofloxacin, amikacin, imipenem, and colistin at four-fold the MIC, while tigecycline-resistant mutants generated with a frequency between $1.2 \times 10^{-8}$ and $9.0 \times 10^{-8}$ for all tested isolates (see Additional file 2).

Search for efflux genes showed that all isolates were positive for the adeB and adeJ genes and negative for both adeE and $a d e Y$ genes, irrespective of their resistance or susceptibility profile. All of the isolates were positive for the twocomponent regulatory system adeRS.

\section{Discussion}

In this work we investigated tigecycline interactions with various antimicrobials by a two-step approach, involving 
Table 2: Chequerboard results obtained with tigecycline in combination with seven antibiotics in 24 A. baumannii isolates

\begin{tabular}{|c|c|c|c|c|c|c|c|}
\hline \multirow[b]{2}{*}{ Study code } & \multicolumn{7}{|c|}{ Effect ( $\mathrm{FICl}$ value) of TIG in combination with a } \\
\hline & LVX & TZP & AMK & IPM & RIF & SAM & CS \\
\hline 5 & Sy $(0.31)$ & $\ln (2.03)$ & An (4.06) & $\ln (0.75)$ & $\ln (0.62)$ & $\ln (1.25)$ & $\ln (0.62)$ \\
\hline 11 & Sy $(0.31)$ & $\ln (2.03)$ & Sy $(0.50)$ & $\ln (0.75)$ & $\ln (0.62)$ & $\ln (1.50)$ & $\ln (0.56)$ \\
\hline 16 & Sy $(0.50)$ & $\ln (1.03)$ & $\ln (0.62)$ & $\ln (0.75)$ & $\ln (1.06)$ & $\ln (1.50)$ & Sy $(0.50)$ \\
\hline 28 & $\ln (0.62)$ & $\ln (2.03)$ & An (8.06) & $\ln (0.75)$ & $\ln (1.00)$ & $\ln (1.50)$ & $\ln (0.56)$ \\
\hline 29 & $\ln (0.75)$ & $\ln (2.06)$ & $\ln (0.75)$ & $\ln (0.75)$ & $\ln (1.00)$ & $\ln (1.50)$ & $\ln (1.12)$ \\
\hline 32 & $\ln (1.12)$ & $\ln (2.06)$ & $\ln (1.25)$ & $\ln (0.75)$ & $\ln (1.00)$ & $\ln (1.50)$ & $\ln (0.62)$ \\
\hline 50 & $\ln (0.75)$ & An (8.03) & $\ln (0.75)$ & $\ln (0.75)$ & $\ln (0.75)$ & $\ln (2.50)$ & $\ln (0.56)^{b}$ \\
\hline 62 & $\ln (1.00)$ & $\ln (1.00)$ & $\ln (1.00)$ & Sy $(0.37)$ & $\ln (0.62)$ & $\ln (1.00)$ & $\ln (0.62)$ \\
\hline 63 & $\ln (0.56)$ & $\ln (2.03)$ & $\ln (0.56)$ & $\ln (0.75)$ & $\ln (0.75)$ & $\ln (1.25)$ & $\ln (0.56)$ \\
\hline 71 & $\ln (0.75)$ & $\ln (2.03)$ & Sy $(0.50)$ & $\ln (0.75)$ & $\ln (0.56)$ & $\ln (1.25)$ & $\ln (0.56)$ \\
\hline 73 & $\ln (0.75)$ & An (4.06) & $\ln (1.12)$ & $\ln (1.12)$ & $\ln (1.50)$ & $\ln (2.50)$ & An (4.25) \\
\hline 75 & Sy $(0.31)$ & $\ln (0.75)$ & $\ln (0.62)$ & $\ln (0.62)$ & $\ln (0.62)$ & $\ln (0.56)$ & Sy $(0.50)$ \\
\hline 80 & $\ln (0.62)$ & An (4.06) & $\ln (0.62)$ & Sy $(0.37)$ & $\ln (1.00)$ & $\ln (1.25)$ & $\ln (0.62)$ \\
\hline 82 & $\ln (0.56)$ & $\ln (0.75)$ & $\ln (2.12)$ & $\ln (1.25)$ & $\ln (0.62)$ & $\ln (0.75)$ & $\ln (1.25)$ \\
\hline 86 & $\ln (1.00)$ & An (4.06) & $\ln (1.00)$ & $\ln (0.75)$ & $\ln (1.00)$ & $\ln (1.50)$ & $\ln (0.56)$ \\
\hline 87 & $\ln (0.56)$ & $\ln (0.75)$ & $\ln (0.56)$ & $\ln (0.75)$ & $\ln (2.12)$ & $\ln (0.75)$ & $\ln (1.12)$ \\
\hline 88 & $\ln (0.62)$ & $\ln (1.00)$ & $A n(4.03)$ & $\ln (0.75)$ & $\ln (0.75)$ & $\ln (0.62)$ & $\ln (0.75)$ \\
\hline 89 & $\ln (0.62)$ & $\ln (1.50)$ & $\ln (1.06)$ & $\ln (0.75)$ & $\ln (0.75)$ & $\ln (0.75)$ & $\ln (1.12)$ \\
\hline 93 & $\ln (0.56)$ & An (4.06) & $\ln (2.06)$ & $\ln (1.00)$ & An (4.25) & $\ln (1.00)$ & $\ln (0.62)$ \\
\hline 100 & $\ln (0.56)$ & $\ln (2.03)$ & $\ln (1.00)$ & $\ln (1.00)$ & $\ln (1.00)$ & $\ln (0.75)$ & $\ln (0.62)$ \\
\hline 105 & $\ln (2.03)$ & An (4.03) & $\ln (0.75)$ & $\ln (2.50)$ & $\ln (2.50)$ & An (4.25) & $\ln (2.25)$ \\
\hline 115 & $\ln (1.06)$ & An (4.06) & $\ln (2.25)$ & $\ln (1.50)$ & $\ln (1.50)$ & $\ln (2.25)$ & $\ln (1.06)$ \\
\hline RUH I34 & $\ln (1.50)^{c}$ & An (4.I2) & $\ln (0.75)$ & $\ln (1.50)^{c}$ & $\ln (1.00)$ & $\ln (2.12)$ & $\ln (1.06)$ \\
\hline RUH 875 & $\ln (1.50)^{c}$ & $\ln (2.06)$ & $\ln (2.12)$ & $\ln (1.00)^{c}$ & $\ln (1.00)$ & $\ln (2.06)$ & $\ln (2.12)$ \\
\hline
\end{tabular}

a Synergistic and antagonistic interactions are in bold and italics, respectively.

b The $\mathrm{FICl}$ value of the tigecycline/colistin combination has been calculated considering a MIC value of $32 \mathrm{mg} / \mathrm{L}$ for colistin [see Additional file I]. ${ }^{c} \mathrm{FICl}$ value of tigecycline in combination with levofloxacin and imipenem has been calculated considering a MIC value of $0.125 \mathrm{mg} / \mathrm{L}$ for both levofloxacin and imipenem [see Additional file I].

preliminary chequerboard screening and subsequent time-kill assays. The chequerboard is an easy to perform, high-throughput method which provides single timepoint evidence of bacterial growth inhibition, and generally results in an overestimate of synergistic interactions [22]. For these reasons, all effective combinations inferred from chequerboard analysis were reassessed by time-kill assays. Although time consuming and cumbersome, the time-kill assays provide a dynamic picture of antibiotic action over time [40]. Hence, only combinations showing synergy in both assays were interpreted as authentic synergistic interactions.

While chequerboard screening provided synergistic results for the combinations tigecycline/levofloxacin, tigecycline/ amikacin, tigecycline/imipenem and tigecycline/colistin in 7 out of 24 isolates, time-kill kinetics confirmed synergism in only 5 out of 7 isolates, 4 of which were resistant

Table 3: MIC values for individual antibiotics alone (as determined by the broth microdilution method) and in effective synergistic combination with tigecycline (as determined by the chequerboard method) for seven $A$. baumannii isolates

\begin{tabular}{|c|c|c|c|c|c|c|c|c|c|}
\hline \multirow[b]{2}{*}{ Study code } & \multicolumn{9}{|c|}{ MIC (mg/L) a } \\
\hline & TIG & LVX & AMK & IPM & CS & TIG/LVX & TIG/AMK & TIG/IPM & TIG/CS \\
\hline 5 & 4 & 16 & 64 & 16 & 0.25 & $0.25 / 4$ & NS & NS & NS \\
\hline II & 4 & 16 & 64 & 16 & 0.5 & $0.25 / 4$ & $1 / 16$ & NS & NS \\
\hline 16 & 8 & 16 & 128 & 16 & 0.125 & $2 / 4$ & NS & NS & $2 / 0.03$ \\
\hline 62 & 4 & 8 & 2 & 2 & 0.25 & NS & NS & $0.25 / 0.5$ & NS \\
\hline 71 & 4 & 8 & 256 & 16 & 0.5 & NS & $1 / 64$ & NS & NS \\
\hline 75 & 4 & 16 & 128 & 2 & 0.5 & $0.25 / 4$ & NS & NS & $1 / 0.125$ \\
\hline 80 & 4 & 8 & 128 & 32 & 0.25 & NS & NS & $0.25 / 8$ & NS \\
\hline
\end{tabular}

a Breakpoint criteria are provided in Additional file I. 
A

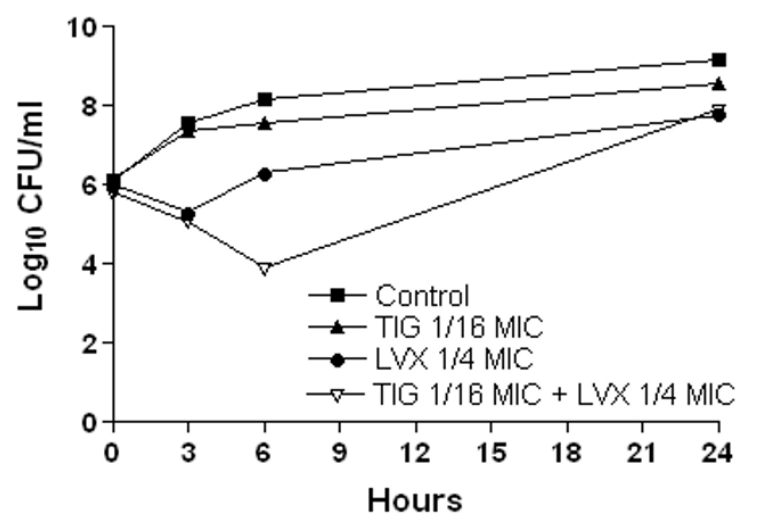

C

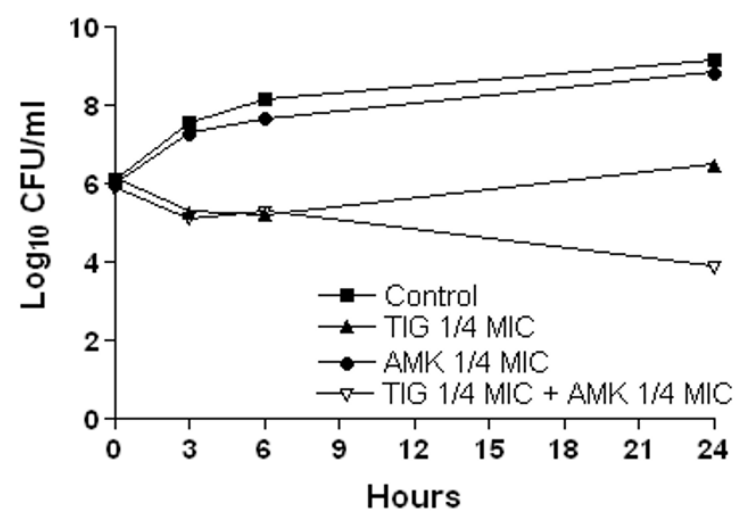

$E$

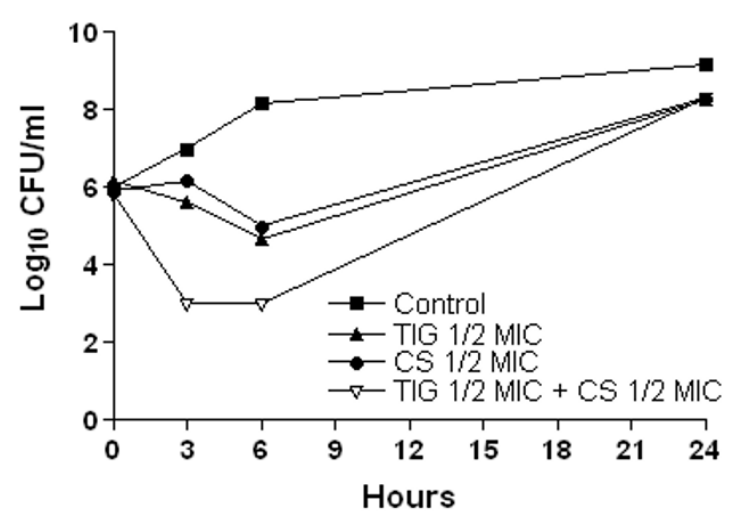

B

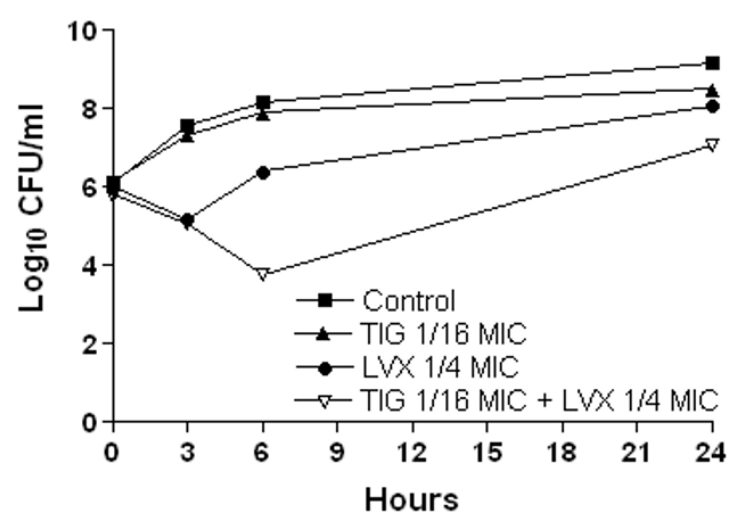

D

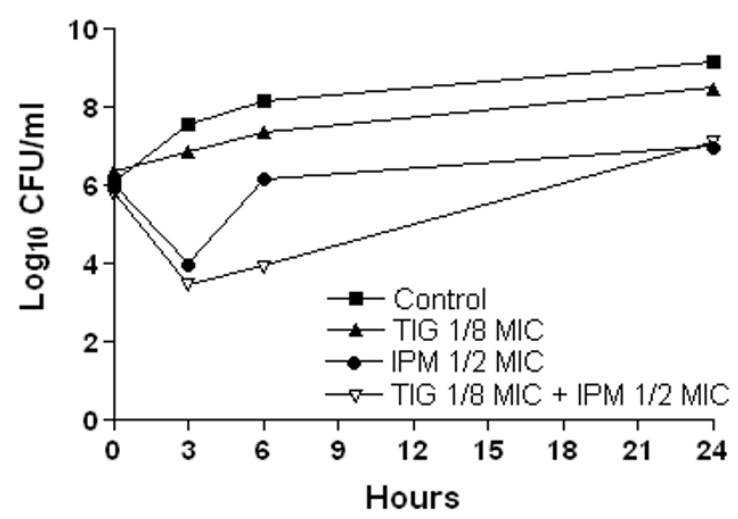

$\mathrm{F}$

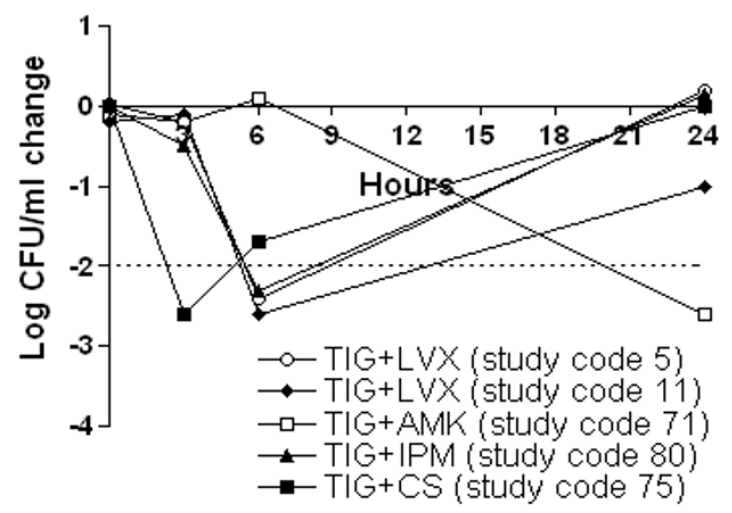

Figure I

Time-kill kinetics for confirmed synergistic interactions. (A) TIG/LVX, study code 5; (B) TIG/LVX, study code II; (C) TIG/AMK, study code 7I, (D) TIG/IPM, study code 80; (E) TIG/CS, study code 75; (F) Comparison of quantitative change in $\mathrm{CFU} / \mathrm{ml}$, relative to the most active constituent, for the synergistic interactions. The drug concentrations are as follows: TIG/ LVX, 0.25 and $4 \mathrm{mg} / \mathrm{L}$, respectively (study codes 5 and II); TIG/AMK, I and $64 \mathrm{mg} / \mathrm{L}$, respectively (study code 7I); TIG/IPM, 0.5 and $16 \mathrm{mg} / \mathrm{L}$, respectively (study code 80 ); TIG/CS, 2 and $0.25 \mathrm{mg} / \mathrm{L}$, respectively (study code 75). The dotted line denotes the threshold value to define synergy. Panels show one representative experiment of three replicates. 
to carbapenems and belonged to type 1 (related to the European clonal lineage II) [26]. The different synergistic activities observed in $A$. baumannii isolates sharing the same epidemiological type [26] probably reflect the variable expression of different resistance determinants. This poses the need to test synergistic interactions even in case of clonal isolates characterized by identical genetic fingerprint and resistance profile.

Three out of five tigecycline synergistic concentrations observed in this study with time-kill assays exceed the maximum plasmatic concentration of tigecycline $(0.38$ $\mathrm{mg} / \mathrm{L}$ ) achievable with a standard dosage [41]. However, thanks to its pharmacodynamic properties, tigecycline is rapidly distributed into tissues resulting in up to 78-fold higher tissue concentrations, compared to plasma $[42,43]$. These considerations suggest a clinical usefulness for some of the synergistic combinations here detected for tigecycline.

We identified one isolate (study code 71) showing tigecycline/amikacin synergistic interaction at $24 \mathrm{~h}$ ( 1 and 64 $\mathrm{mg} / \mathrm{L}$ for tigecycline and amikacin, respectively). This strain was resistant to amikacin (MIC $=256 \mathrm{mg} / \mathrm{L}$ ) and showed an intermediate resistance to tigecycline $(\mathrm{MIC}=4$ $\mathrm{mg} / \mathrm{L})$. Although the synergistic concentration for amikacin $(64 \mathrm{mg} / \mathrm{L})$ is significantly above the threshold achievable in clinical treatments with a multi daily dosing regimen (20-30 mg/L), higher concentrations (65-75 $\mathrm{mg} / \mathrm{L}$ ) can be achieved with a single amikacin daily dose [44].

In vitro synergistic interactions between tigecycline and colistin have previously been demonstrated by time-kill assays in Klebsiella pneumoniae [45] and in vivo for the treatment of a severe case of MDR Pseudomonas aeruginosa osteomyelitis [46]. Although several studies have reported clinical efficacy of colistin [7,8], the synergistic effect of tigecycline/colistin combination has never been demonstrated in A. baumannii by time-kill analysis. Here, we showed for one A. baumannii isolate (study code 75) a synergistic effect at $3 \mathrm{~h}$ of incubation for tigecycline/colistin combination ( 2 and $0.25 \mathrm{mg} / \mathrm{L}$ for tigecycline and colistin, respectively), with a subsequent re-growth within 24 h. This strain was susceptible to colistin (MIC $=0.5 \mathrm{mg} / \mathrm{L})$ and intermediate resistant to tigecycline $(\mathrm{MIC}=4 \mathrm{mg} / \mathrm{L})$. Notably, the colistin synergistic concentration is significantly below the serum concentration achievable after standard dosing regimen $(5-6 \mathrm{mg} / \mathrm{L})[44,47]$. As noted by various authors [48-50], colistin causes permeabilisation of the bacterial outer membrane, which would allow enhanced penetration by and activity of the other antibiotic in combination. The tigecycline/colistin synergistic interaction could therefore have an impact in clinical practice by reducing the therapeutic dosage of colistin, and hence the risk of collateral effects which currently represent a major limitation to its clinical use $[7,8]$.

We also demonstrated a synergistic interaction for the combination tigecycline/imipenem $(0.5$ and $16 \mathrm{mg} / \mathrm{L}$ for tigecycline and imipenem, respectively) in one A. baumannii isolate (study code 80 ), belonging to the epidemic type 1 , and carrying the $b l a_{\text {OXA-58 }}$ gene [26]. It is of note that the serum concentration achievable during imipenem treatment is $20 \mathrm{mg} / \mathrm{L}$ [47]. Thus, the synergistic interaction tigecycline/imipenem, which has never been described before for A. baumannii, could represent a valid therapeutic option to combat the increasingly frequent A. baumannii isolates resistant to both these drugs.

Resistance to quinolones is widespread among MDR A. baumannii strains [51]. In this study, a high percentage of A. baumannii isolates were resistant to levofloxacin as a single agent. Here we report for the first time a synergistic interaction between tigecycline and levofloxacin $(0.25$ and $4 \mathrm{mg} / \mathrm{L}$ respectively) for 2 A. baumannii isolates, at 6 $\mathrm{h}$ of incubation. These strains showed full resistance to levofloxacin ( $\mathrm{MIC}=16 \mathrm{mg} / \mathrm{L}$ ) and intermediate resistance to tigecycline $(\mathrm{MIC}=4 \mathrm{mg} / \mathrm{L})$. Also in this instance, the levofloxacin synergistic concentration is below the maximum serum concentration (5.9 mg/L) [52].

Even if no undesirable antagonistic combinations were confirmed in this study by time-kill assay, we detected a decreased antimicrobial efficacy for the tigecycline/piperacillin-tazobactam combination, compared to the antimicrobial efficacy of piperacillin-tazobactam alone (data not shown). This result is worrying considering that tigecycline/piperacillin-tazobactam combination therapy is often given empirically, without the support of in vitro interaction assays.

The molecular mechanisms of synergy between tigecycline and the various antibiotics deserve further investigation. Overexpression of the AdeABC efflux pump has been demonstrated in tigecycline resistant $A$. baumannii isolates [53], and our results indicate that all A. baumannii isolates tested carry the adeABC/adeIJK genes, suggesting that their variable expression level - but not their presence per se could contribute to the extent of resistance. We also showed that adeDE is not present in A. baumannii, in agreement with previous studies [38,39].

The regrowth after 24 h observed in time-kill experiments for all confirmed synergistic combination, except for tigecycline/amikacin, could reflect the labile nature in solution of tigecycline due to oxidative degradation (Wyeth Research, unpublished data) and/or the tendency of $A$. baumannii strains to induce resistance on exposure to antimicrobial agents, especially at sub-MIC concentrations. At 
present, we are unable to check the tigecycline levels and therefore we cannot determine if tigecycline was degraded, at least partially, during the experimental time course.

Determination of mutation frequencies for resistance to levofloxacin, amikacin, imipenem, colistin, and tigecycline at four-fold the MIC failed to detect any hypermutator phenotype for all isolates showing synergy in time-kill assays, irrespective of regrowth. Moreover, the mutation frequency toward resistance to tigecycline $\left(\sim 5 \times 10^{-8}\right)$ or other antibiotics $\left(<10^{-8}\right)$ is incompatible with the observed regrowth kinetics (Figure 1 ). Hence, we can only speculate that regrowth was due to different response of isolates to antibiotic-induced overexpression of broadspecificity multidrug efflux systems, like AdeABC and AdeIJK [16,37-39], rather than hypermutability. According to this hypothesis, the tigecycline/amikacin interaction may have prevented the expression of efflux-based resistance by a still undefined mechanism, ultimately resulting in more effective synergism. In fact, a recent study on tigecycline/amikacin synergistic interactions in A. baumannii demonstrated the suppression of regrowth at $24 \mathrm{~h}$ for this particular antibiotic combination, in full agreement with our findings [25].

Further studies are needed to elucidate the molecular mechanisms responsible for synergistic interactions with tigecycline and to explore their therapeutic potential. It will also be necessary to combine in vitro findings with additional pharmacokinetic and pharmacodynamic data in order to provide more meaningful prediction of the in vivo efficacy of synergistic combinations in clinical practice. Lastly, in vitro synergy testing of tigecycline combinations is recommended prior to starting any combined therapy for treatment of infections sustained by MDR and pan-resistant A. baumannii.

\section{Abbreviations}

AMK: amikacin; CS: colistin; IPM: imipenem; LVX: levofloxacin; RIF: rifampicin; SAM: ampicillin-sulbactam; TIG: tigecycline; TZP: piperacillin-tazobactam; Sy: synergy; In: indifference; An: antagonism; NS: not synergic; S: susceptible; I: intermediate; R: resistant.

\section{Competing interests}

The authors declare that they have no competing interests.

\section{Authors' contributions}

LP and SD performed the susceptibility tests, the molecular genetics studies and drafted part of the manuscript. AC participated in the design of the study, provided clinical interpretation of susceptibility data, and drafted part of the manuscript. NP and PV conceived of the study, participated in its design and coordination, and critically revised the draft manuscript. All authors read and approved the final manuscript.

\section{Additional material}

\section{Additional file 1}

Distribution of MIC values and antibiotic susceptibility profile for 24 A. baumannii isolates. The data provided are MIC values and antibiotic susceptibility categories $(S, I, R)$ for all strains analysed in this study. Click here for file

[http://www.biomedcentral.com/content/supplementary/14760711-8-18-S1.doc]

\section{Additional file 2}

Mutation frequency for resistance to antibiotics at four-fold the MIC. The data provided are mutation frequencies for resistance to TIG, LVX, AMK, IPM and CS for strains showing authentic synergism according to the definition provided in Methods.

Click here for file

[http://www.biomedcentral.com/content/supplementary/14760711-8-18-S2.doc]

\section{Acknowledgements}

Wyeth-Ayerst (Collegeville, Pennsylvania, USA) kindly provided laboratory-grade tigecycline. This research was supported by the INMI "L. Spallanzani" - Ricerca Corrente 2007 grant to P.V.

\section{References}

I. Munoz-Price LS, Weinstein RA: Acinetobacter infection. $N$ Engl J Med 2008, 358: $|27|-\mid 281$.

2. Abbo A, Navon-Venezia S, Hammer-Muntz O, Krichali T, SiegmanIgra Y, Carmeli Y: Multidrug-resistant Acinetobacter baumannii. Emerg Infect Dis 2005, I I:22-29.

3. Poirel L, Nordmann P: Carbapenem resistance in Acinetobacter baumannii : mechanisms and epidemiology. Clin Microbiol Infect 2006, I 2:826-836.

4. Nemec A, Krízová L, Maixnerová M, Diancourt L, Reijden TJ van der, Brisse S, Broek $P$ van den, Dijkshoorn L: Emergence of carbapenem resistance in Acinetobacter baumannii in the Czech Republic is associated with the spread of multidrug-resistant strains of European clone II. J Antimicrob Chemother 2008, 62:484-489.

5. Coelho JM, Turton JF, Kaufmann ME, Glover J, Woodford N, Warner M, Palepou MF, Pike R, Pitt TL, Patel BC, Livermore DM: Occurrence of carbapenem-resistant Acinetobacter baumannii clones at multiple hospitals in London and Southeast England. J Clin Microbiol 2006, 44:3623-3627.

6. Li J, Rayner CR, Nation RL, Owen RJ, Spelman D, Tan KE, Liolios L: Heteroresistance to colistin in multidrug-resistant Acinetobacter baumannii. Antimicrob Agents Chemother 2006, 50:2946-2950.

7. Falagas ME, Kasiakou SK: Colistin: the revival of polymyxins for the management of multidrug-resistant gram-negative bacterial infections. Clin Infect Dis 2005, 40:1333-I34I.

8. Petrosillo N, loannidou E, Falagas ME: Colistin monotherapy vs. combination therapy: evidence from microbiological, animal and clinical studies. Clin Microbiol Infect 2008, 14:8I6-827.

9. Henwood CJ, Gatward T, Warner M, James D, Stockdale MW, Spence RP, Towner KJ, Livermore DM, Woodford N: Antibiotic resistance among clinical isolates of Acinetobacter in the UK, and in vitro evaluation of tigecycline (GAR-936). J Antimicrob Chemother 2002, 49:479-487.

10. Pachón-lbáñez ME, Jiménez-Mejías ME, Pichardo C, Llanos AC, Pachón J: Activity of tigecycline (GAR-936) against Acineto- 
bacter baumannii strains, including those resistant to imipenem. Antimicrob Agents Chemother 2004, 48:4479-448I.

II. Navon-Venezia S, Leavitt A, Carmeli Y: High tigecycline resistance in multidrug-resistant Acinetobacter baumannii. J Antimicrob Chemother 2007, 59:772-774.

12. Insa R, Cercenado E, Goyanes MJ, Morente A, Bouza E: In vitro activity of tigecycline against clinical isolates of Acinetobacter baumannii and Stenotrophomonas maltophilia. J Antimicrob Chemother 2007, 59:583-585.

13. Capone A, D'Arezzo S, Visca P, Petrosillo N: In vitro activity of tigecycline against multidrug-resistant Acinetobacter baumannii. J Antimicrob Chemother 2008, 62:422-423.

I4. Lolans K, Rice TW, Munoz-Price LS, Quinn JP: Multicity outbreak of carbapenem-resistant Acinetobacter baumannii isolates producing the carbapenemase OXA-40. Antimicrob Agents Chemother 2006, 50:294 I-2945.

15. Zhang YY, Zhou L, Zhu DM, Wu PC, Hu FP, Wu WH, Wang F: In vitro activities of tigecycline against clinical isolates from Shanghai, China. Diagn Microbiol Infect Dis 2004, 50:267-28I.

16. Peleg AY, Adams J, Paterson DL: Tigecycline efflux as a mechanism for nonsusceptibility in Acinetobacter baumannii. Antimicrob Agents Chemother 2007, 5 I:2065-2069.

17. Karageorgopoulos DE, Kelesidis T, Kelesidis I, Falagas ME: Tigecycline for the treatment of multidrug-resistant (including carbapenem-resistant) Acinetobacter infections: a review of the scientific evidence. J Antimicrob Chemother 2008, 62:45-55.

18. Reid GE, Grim SA, Aldeza CA, Janda WM, Clark NM: Rapid development of Acinetobacter baumannii resistance to tigecycline. Pharmacotherapy 2007, 27: I| 98-I201.

19. Perez F, Hujer AM, Hujer KM, Decker BK, Rather PN, Bonomo RA: Global challenge of multidrug-resistant Acinetobacter baumannii. Antimicrob Agents Chemother 2007, 5 I:347I-3484

20. Sands M, McCarter $Y$, Sanchez $W$ : Synergy testing of multidrug resistant Acinetobacter baumanii against tigecycline and polymyxin using an E-test methodology. Eur J Clin Microbiol Infect Dis. 2007, 26(7):52I-522.

21. Scheetz MH, Qi C, Warren JR, Postelnick MJ, Zembower T, Obias A, Noskin GA: In vitro activities of various antimicrobials alone and in combination with tigecycline against carbapenemintermediate or -resistant Acinetobacter baumannii. Antimicrob Agents Chemother 2007, 51:1621-1626.

22. Petersen PJ, Labthavikul P, Jones $\mathrm{CH}$, Bradford PA: In vitro antibacterial activities of tigecycline in combination with other antimicrobial agents determined by chequerboard and time-kill kinetic analysis. I Antimicrob Chemother 2006, 57:573-576.

23. Taccone FS, Rodriguez-Villalobos H, De Backer D, De Moor V, Deviere J, Vincent JL, Jacobs F: Successful treatment of septic shock due to pan-resistant Acinetobacter baumannii using combined antimicrobial therapy including tigecycline. Eur J Clin Microbiol Infect Dis 2006, 25:257-260.

24. Leclerc T, Perez JP, Debien B, Clapson P, Lenoir B: Treatment of a septic shock due to multiresistant Acinetobacter baumannii with tigecycline in combination. Ann Fr Anesth Reanim 2007, 26:1056-1058

25. Moland ES, Craft DW, Hong SG, Kim SY, Hachmeister L, Sayed SD, Thomson KS: In vitro activity of tigecycline against multidrugresistant Acinetobacter baumannii and selection of tigecycline-amikacin synergy. Antimicrob Agents Chemother 2008, 52:2940-2942.

26. D'Arezzo S, Capone A, Petrosillo N, Visca P: Epidemic multidrug resistant Acinetobacter baumannii related to European clonal types I and II in Rome (Italy). Clin Microbiol Infect 2009.

27. Longo B, Pantosti A, Luzzi I, Placanica P, Gallo S, Tarasi A, Di Sora F, Monaco M, Dionisi AM, Volpe I, Montella F, Cassone A, Rezza G: An outbreak of Acinetobacter baumannii in an intensive care unit: epidemiological and molecular findings. J Hosp Infect 2006, 64:303-305.

28. lacono M, Villa L, Fortini $D$, Bordoni R, Imperi F, Bonnal RJ, SicheritzPonten T, De Bellis G, Visca P, Cassone A, Carattoli A: Wholegenome pyrosequencing of an epidemic multidrug-resistant Acinetobacter baumannii strain belonging to the European clone II group. Antimicrob Agents Chemother 2008, 52:2616-2625.

29. Nemec A, Dijkshoorn L, van der Reijden TJ: Long-term predominance of two pan-European clones among multi-resistant Acinetobacter baumannii strains in the Czech Republic. I Med Microbiol 2004, 53:| 147-I53.
30. Falagas ME, Koletsi PK, Bliziotis IA: The diversity of definitions of multidrug-resistant (MDR) and pandrug-resistant (PDR) Acinetobacter baumannii and Pseudomonas aeruginosa. J Med Microbiol 2006, 55:1619-1629.

31. Paterson DL: The epidemiological profile of infections with multidrug-resistant Pseudomonas aeruginosa and Acinetobacter species. Clin Infect Dis 2006, 43(Suppl 2):43-48.

32. Clinical and Laboratory Standard Institute: Performance standards for antimicrobial susceptibility testing, Seventeenth informational supplement. CLSI document MIO0 SI7. Wayne PA. USA 2007.

33. Gales AC, Reis AO, Jones RN: Contemporary assessment of antimicrobial susceptibility testing methods for polymyxin $B$ and colistin: review of available interpretative criteria and quality control guidelines. / Clin Microbiol 200I, 39:183-I90.

34. Hogg GM, Barr JG, Webb CH: In-vitro activity of the combination of colistin and rifampicin against multidrug-resistant strains of Acinetobacter baumannii. J Antimicrob Chemother 1998, 4I:494-495.

35. Miller K, O'Neill AJ, Chopra I: Response of Escherichia coli hypermutators to selection pressure with antimicrobial agents from different classes. J Antimicrob Chemother 2002, 49:925-934.

36. Prunier AL, Malbruny B, Laurans M, Brouard J, Duhamel JF, Leclercq $R$ : High rate of macrolide resistance in Staphylococcus aureus strains from patients with cystic fibrosis reveals high proportions of hypermutable strains. J Infect Dis 2003, 187:1709-1716.

37. Magnet S, Courvalin P, Lambert T: Resistance-nodulation-cell division-type efflux pump involved in aminoglycoside resistance in Acinetobacter baumannii strain BM4454. Antimicrob Agents Chemother 200I, 45:3375-3380.

38. Lin L, Ling BD, Li XZ: Distribution of the multidrug efflux pump genes, $a d e A B C$, $a d e D E$ and $a d e l J K$, and class $I$ integron genes in multiple-antimicrobial-resistant clinical isolates of Acinetobacter baumannii-Acinetobacter calcoaceticus complex. Int J Antimicrob Agents 2009, 33:27-32.

39. Chu YW, Chau SL, Houang ET: Presence of active efflux systems AdeABC, AdeDE and AdeXYZ in different Acinetobacter genomic DNA groups. J Med Microbiol 2006, 55:477-478.

40. Entenza JM, Moreillon P: Tigecycline in combination with other antimicrobials: a review of in vitro, animal and case report studies. Int J Antimicrob Agents 2009 in press.

4I. Meagher AK, Ambrose PG, Grasela TH, Ellis-Grosse E): The pharmacokinetic and pharmacodynamic profile of tigecycline. Clin Infect Dis 2005, 4 I (Suppl 5):333-340.

42. Rodvold KA, Gotfried MH, Cwik M, Korth-Bradley JM, Dukart G, Ellis-Grosse E): Serum, tissue and body fluid concentrations of tigecycline after a single $100 \mathrm{mg}$ dose. J Antimicrob Chemother 2006, 58: |22I-I229.

43. Peterson LR: A review of tigecycline - the first glycylcycline. Int J Antimicrob Agents 2008, 32(Suppl 4):215-222.

44. Cunha BA: New uses for older antibiotics: nitrofurantoin, amikacin, colistin, polymyxin B, doxycycline, and minocycline revisited. Med Clin North Am 2006, 90: 1089-I 107.

45. Cobo J, Morosini MI, Pintado V, Tato M, Samaranch N, Baquero F, Cantón R: Use of tigecycline for the treatment of prolonged bacteremia due to a multiresistant VIM-I and SHV-I 2 betalactamase-producing Klebsiella pneumoniae epidemic clone. Diagn Microbiol Infect Dis 2008, 60:3 19-322.

46. Stanzani M, Tumietto F, Giannini MB, Bianchi G, Nanetti A, Vianelli N Arpinati M, Giovannini M, Bonifazi F, Bandini G, Baccarani M: Successful treatment of multi-resistant Pseudomonas aeruginosa osteomyelitis after allogeneic bone marrow transplantation with a combination of colistin and tigecycline. J Med Microbiol 2007, 56:1692-1695.

47. Tripodi MF, Durante-Mangoni E, Fortunato R, Utili R, Zarrilli R: Comparative activities of colistin, rifampicin, imipenem and sulbactam/ampicillin alone or in combination against epidemic multidrug-resistant Acinetobacter baumannii isolates producing OXA-58 carbapenemases. Int J Antimicrob Agents 2007, 30:537-540.

48. Timurkaynak F, Can F, Azap OK, Demirbilek M, Arslan H, Karaman SO: In vitro activities of non-traditional antimicrobials alone or in combination against multidrug-resistant strains of Pseudomonas aeruginosa and Acinetobacter baumannii isolated from intensive care units. Int / Antimicrob Agents 2006, 27:224-228. 
49. Tascini C, Gemignani G, Ferranti S, Tagliaferri E, Leonildi A, Lucarini A, Menichetti F: Microbiological activity and clinical efficacy of a colistin and rifampin combination in multidrug-resistant Pseudomonas aeruginosa infections. I Chemother 2004, 16:282-287.

50. Giamarellos-Bourboulis E], Karnesis L, Giamarellou H: Synergy of colistin with rifampin and trimethoprim/sulfamethoxazole on multidrug-resistant Stenotrophomonas maltophilia. Diagn Microbiol Infect Dis 2002, 44:259-263.

5I. Hoban DJ, Bouchillon SK, Dowzicky MJ: Antimicrobial susceptibility of extended-spectrum beta-lactamase producers and multidrug-resistant Acinetobacter baumannii throughout the United States and comparative in vitro activity of tigecycline, a new glycylcycline antimicrobial. Diagn Microbiol Infect Dis 2007, 57:423-428.

52. Geerdes-Fenge HF, Wiedersich A, Wagner S, Lehr KH, Koeppe P, Lode $\mathrm{H}$ : Levofloxacin pharmacokinetics and serum bactericidal activities against five enterobacterial species. Antimicrob Agents Chemother 2000, 44:3478-3480.

53. Ruzin $A$, Keeney $D$, Bradford PA: AdeABC multidrug efflux pump is associated with decreased susceptibility to tigecycline in Acinetobacter calcoaceticus-Acinetobacter baumannii complex. J Antimicrob Chemother 2007, 59:100I-1004.

Publish with Bio Med Central and every scientist can read your work free of charge

"BioMed Central will be the most significant development for disseminating the results of biomedical research in our lifetime. "

Sir Paul Nurse, Cancer Research UK

Your research papers will be:

- available free of charge to the entire biomedical community

- peer reviewed and published immediately upon acceptance

- cited in PubMed and archived on PubMed Central

- yours - you keep the copyright 